\title{
Pelvic exenteration for recurrent rectal cancer involving the small bowel and abdominal wall - case report
}

\author{
Bogdan Filip*, lonut Hutanu, Maria Gabriela Anitei, Iulian Radu, Viorel Scripcariu \\ $1^{\text {st }}$ Surgical Unit, Regional Institute of Oncology lasi, Department of Surgery, "Grigore T. Popa", \\ University of Medicine and Pharmacy lasi, Romania
}

\begin{abstract}
Pelvic exenteration represents a radical intervention performed on highly selected cases for locally advanced pelvic (gynecologic and digestive) tumours. Due to the high complexity of the intervention and of the impact of the neoplastic disease on the patient this intervention is accompanied by a high degree of postoperative morbidity. We hereby present the case of a patient with recurrent rectal cancer involving the bladder, small bowel and anterior abdominal wall in which a supralevatorial pelvectomy was performed. Although it was performed on a patient with infected tumour, the postoperative course of the patient was uneventful; the patient is disease-free at 6 months after pelvectomy. Although pelvic exenteration is associated with increased morbidity, many patients with recurrence after rectal resection that undergo this intervention can have increased disease-free survival.
\end{abstract}

Keywords: pelvic exenteration, rectal cancer, morbidity

\section{Introduction}

Pelvic exenteration represents a radical surgical technique used for central recurrences after pelvic tumours in patients who were submitted to radiation therapy. It also can be performed as a palliative procedure for local control of the disease (tumours with severe fistulas or medically uncontrolled symptoms).

This technique was first described in 1948 by Brunschwig in a patient with advanced cervical cancer and included total resection of pelvic viscera (bladder, uterus and rectum)

Received: March 2014; Accepted after review: April 2014; Published: June 2014

${ }^{*}$ Corresponding author: Filip Bogdan, $1^{\text {st }}$ Department of Surgical Unit, Regional Institute of Oncology lasi, University of Medicine and Pharmacy „Grigore T. Popa”, lasi, Romania.Email: bfilip79@yahoo.com ended with a terminal colostomy and urinary conduit [1]. Due to the high mortality and morbidity this technique was modified over the years, and it was described a Posterior Pelvic Exenteration (resection of rectum, uterus and posterior vaginal wall), Anterior Pelvic Exenteration (resection of bladder and uterus) and Supralevator Pelvic Exenteration (resection of pelvic organs at the level of levator muscle preserving the urogenital diaphragm) [2]. Further developments included a colo-anal anastomosis and orthotopic neobladder construction.

Surgical technique refinement and improvement of critical care of the patient have dropped the postoperative mortality from 18 to $25 \%$ in the first series to less than $10 \%$ in the newest series [3], with a postoperative morbidity between 38 to $65 \%$ [4]. The functional and psychological consequences of this radical intervention can be important 
especially in a patient with a good oncological prognosis.

Local recurrence after curative surgical resection for rectal cancer occurs in 4 to $33 \%$, in many of those cases local recurrence is diagnosed in advanced cases due to the lack of symptoms [5]. Resection of local recurrence is suitable in patients with a good general status, without metastases and who are not suitable for radiotherapy. After curative resection 5 year survival rates are between 30 to $40 \%$.

\section{Case report}

We hereby present the case of a 63 yearold male patient with a large (10 by $15 \mathrm{~cm}$ ) ulcerated abdominal tumour at the level of hypogastrium (Fig. 1).

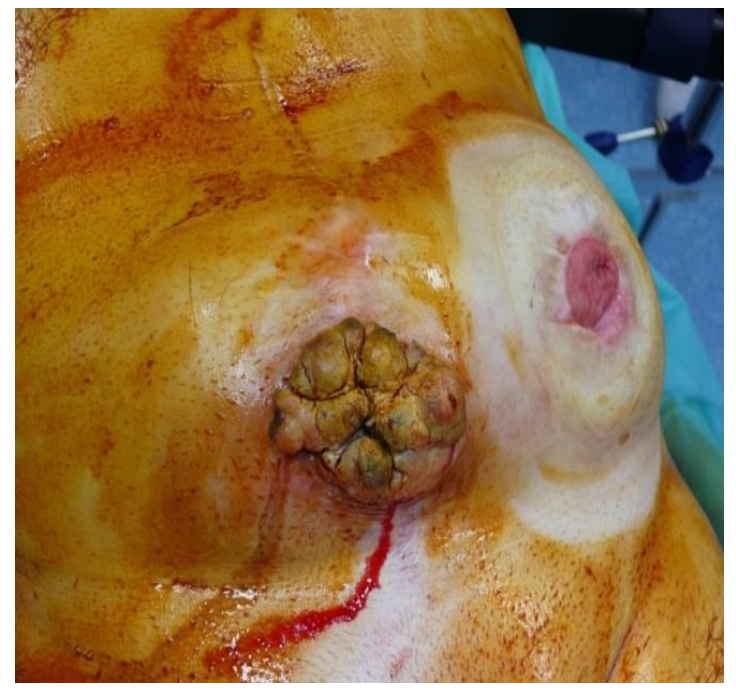

Fig. 1. Ulcerated abdominal tumour at the level of hypogastrium

The patient was diagnosed in July 2010 with a locally advanced tumour at the level of colorectal junction after a laparotomy for acute bowel obstruction when a lateral colostomy was performed. The patient was then submitted to Cisplatin and 5-FU based chemotherapy and six months later was performed a R0 resection (Hartmann procedure with partial cystectomy and small bowel resection). The pathological exam showed a moderate differentiated rectal cancer involving the bladder wall and an ileal loop. The patient was submitted to an oral chemotherapy regiment with Capecitabine and it was lost form regular postoperative observation.

In 2012 was diagnosed with local recurrence of the disease and initially the patient refused any form of treatment, although the tumour recurrence at that time was suitable for resection.

One year after, the patient was admitted in the 1st Surgical Unit of the Regional Institute of Oncology lasi due to the tumor skin ulceration associated with infection and recurrent hematuria.

The patient, with normal weight and blood pressure was otherwise in good health. The blood test showed anemia $(\mathrm{Hb}=7.6 \mathrm{~g} / \mathrm{dl})$, mild leukocytosis, elevated CEA levels. The bacteriological exam from the ulcerated lesion showed the presence of $E$. coli, $P$. aeruginosa and the urine sample was positive for $E$. coli.

Abdominal CT scan showed a large tumour (size 148/100/96 cm) involving the rectal stump, the bladder wall, prostate, mesorectum and ileal small bowel loops (Fig. 2, 3). Also it was noticed on CT scan that the tumour presented central necrosis, gas (as sign of infection) being found around necrotic parts. No major vascular involvement was found. Pulmonary and hepatic metastases were excluded on imaging exams performed.

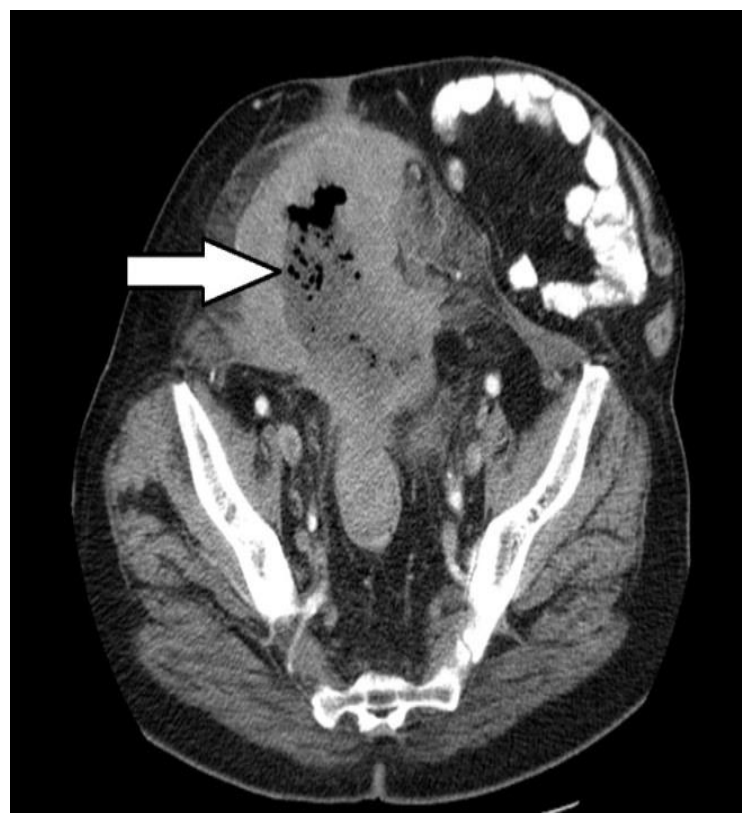

Fig. 2. Abdominal CT scan showing a gross tumour with central necrosis and gas involving the bladder 


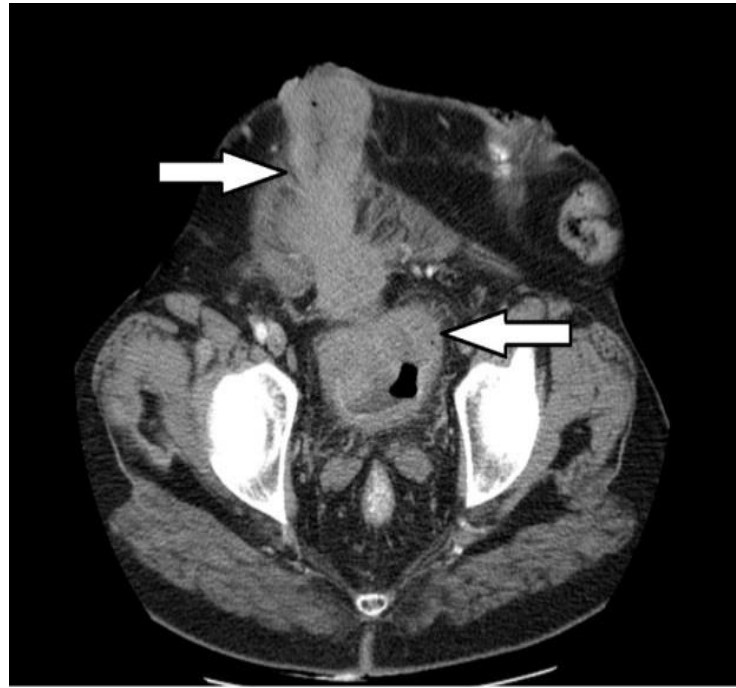

Fig. 3. Involvement of the abdominal wall on abdominal CT scan

Surgery consisted in resection of the tumour together with bladder, prostate, rectal stump and mesorectum (a supralevator pelvic exenteration), a $50 \mathrm{~cm}$ small bowel loop and the part of the abdominal wall involved by the tumour. For the urinary diversion a Bricker technique was performed, the ureteral anastomosis being performed using a tutor (6Fr silicone catheter), removed 14 days after surgery (Fig. 4). Total blood loss on intervention was $400 \mathrm{ml}$, the patient being extubated at the end of the intervention and referred to ICU for further observation.

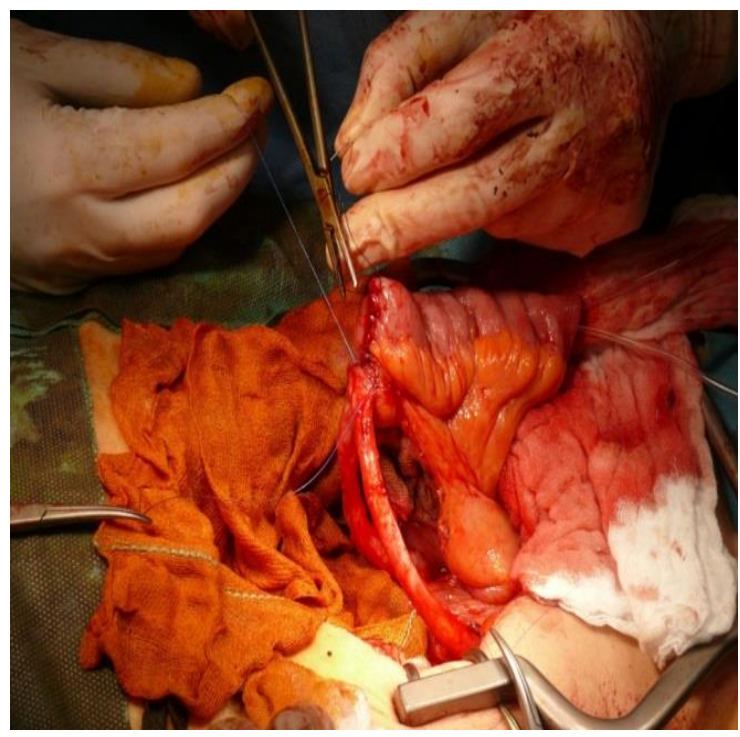

Fig. 4. Final aspect of the reimplantation of the ureters on the ileal loop
Histopathology diagnosed the recurrence as a moderate differentiated rectal adenocarcinoma without lymph node involvement (rpT4bN0). The postoperative course of the patient was uneventful and it was discharged 10 days after surgery. Six months after surgery the patient is in a good general condition and it was submitted to a new regimen of oral Capecitabine.

\section{Discussions}

Pelvic exenteration for recurrent rectal cancer is often performed in tertiary centers by multidisciplinary oncology teams whereby subspecialization and collaboration have been shown to be successful.

Due to the high complexity of the surgical intervention, the early outcome after surgery showed an increased mortality and morbidity, with a continuous descendant trend. Reviewing the latest studies regarding pelvic exenteration for recurrent rectal cancer the mortality varied between 0 and 13\% [6-13]. Late oncological results showed a 5-year survival between 14 and 27\% [9, 12].

Although major developments occurred in diagnosis and oncological therapies, the final decision of proceeding to pelvic exenteration can only be made at the time of laparotomy, difficulties are the impossibility to distinguish between inflammatory and malignant adhesions between organs. The intraoperatory frozen section analysis can mislead the course of surgery due to the fibrosis after radiotherapy. In our reported case the patient presented a recurrence involving the bladder, small bowel, rectal stump and the abdominal wall. The patient accepted the radical intervention due to the ulceration of the tumours with subsequent bacterial colonization and infection. The patient presented a poor quality of life previous to surgery, accepting the potential morbidity associated with urinary diversion.

Multiple studies showed that predictor factors for long-term survival after pelvic exenteration for rectal cancer are tumour stage, presence of nodal metastases and positive surgical margins [10, 14, and 15]. Same factors seem to be involved in long term 
survival after resection for local recurrence. Our patient on initial tumour resection presented negative surgical margins and no positive lymph nodes, although he developed local recurrence.

Certain selected pelvic malignancies, among those, rectal cancer, have favorable biological characteristics that allow them to grow locally without having distant

\section{References:}

1. Brunschwig A.: Complete excision of pelvic viscera for advanced carcinoma; a one-stage abdominoperineal operation with end colostomy and bilateral ureteral implantation into the colon above the colostomy. Cancer, 1948, 1(2):17783. Epub 1948/07/01.

2. Dias AR, Nahas SC: Modified supralevator pelvic exenteration for the treatment of locally advanced rectal cancer with vaginal and uterine invasion. Surg Today, 2013, 43(6):702-4. Epub 2012/08/22.

3. Averette HE, Lichtinger M, Sevin BU, Girtanner RE: Pelvic exenteration: a 15-year experience in a general metropolitan hospital. Am J Obst Gynecol, 1984, 150(2):179-84. Epub 1984/09/15.

4. Soper JT, Berchuck A, Creasman WT, ClarkePearson DL: Pelvic exenteration: factors associated with major surgical morbidity. Gynecol Oncol, 1989, 35(1):93-8. Epub 1989/10/01.

5. Houvenaeghel G. [Conservative surgery and restoration after pelvic exenteration]. Bulletin $d u$ cancer. 2002, 89(12):1061-6. Epub 2003/01/15. Chirurgie de preservation d'organes et de restauration apres exenteration pelvienne.

6. Jimenez RE, Shoup M, Cohen AM, Paty PB, Guillem J, Wong WD: Contemporary outcomes of total pelvic exenteration in the treatment of colorectal cancer. Dis Col Rect, 2003, 46(12):1619-25. Epub 2003/12/12.

7. Meterissian SH, Skibber JM, Giacco GG, elNaggar AK, Hess KR, Rich TA: Pelvic exenteration for locally advanced rectal carcinoma: factors predicting improved survival. Surgery, 1997, 121(5):479-87. Epub 1997/05/01. metastases. This is the main reason which implies a major radical surgery for locally advanced cancers of the pelvis. Although pelvic exenteration is associated with increased morbidity, many patients with recurrence after rectal resection that undergo this intervention can have increased diseasefree survival.

8. Russo P, Ravindran B, Katz J, Paty P, Guillem J, Cohen AM: Urinary diversion after total pelvic exenteration for rectal cancer. Ann Surg Oncol, 1999, 6(8):732-8. Epub 2000/01/06.

9. Ike $H$, Shimada $H$, Ohki $S$, Yamaguchi $S$, Ichikawa Y, Fujii S: Outcome of total pelvic exenteration for locally recurrent rectal cancer. Hepatogastroenterology, 2003, 50(51):700-3. Epub 2003/06/28.

10. Vermaas M, Ferenschild FT, Verhoef $C$ et al.: Total pelvic exenteration for primary locally advanced and locally recurrent rectal cancer. Eur J Surg Oncol, 2007, 33(4):452-8. Epub 2006/10/31

11. Gannon CJ, Zager JS, Chang GJ et al.: Pelvic exenteration affords safe and durable treatment for locally advanced rectal carcinoma. Ann Surg Oncol, 2007, 14(6):1870-7. Epub 2007/04/05.

12. Wells BJ, Stotland $P$, Ko MA et al. Results of an aggressive approach to resection of locally recurrent rectal cancer. Ann Surg Oncol, 2007, 14(2):390-5. Epub 2006/10/26.

13. Domes TS, Colquhoun PH, Taylor B, Izawa JI, House AA, Luke PP: Total pelvic exenteration for rectal cancer: outcomes and prognostic factors. Can J Surg, 2011, 54(6):387-93. Epub 2011/09/24.

14. Ike $H$, Shimada $H$, Yamaguchi S, Ichikawa $Y$, Fujii S, Ohki S: Outcome of total pelvic exenteration for primary rectal cancer. Dis Col Rect, 2003, 46(4):474-80. Epub 2003/04/12.

15. Nakafusa Y, Tanaka T, Tanaka M, Kitajima Y, Sato S, Miyazaki K.: Comparison of multivisceral resection and standard operation for locally advanced colorectal cancer: analysis of prognostic factors for short-term and longterm outcome. Dis Col Rect, 2004, 47(12):205563. Epub 2005/01/20. 